\title{
NOUVELLe
}

\section{Contrôle de l'infection des granulocytes neutrophiles par Helicobacter pylori via I'interaction HopQ-CEACAM}

Margot Draveny ${ }^{1}$, Assmaa Ghali ${ }^{1}$, Oliver Nüsse ${ }^{2}$

\author{
${ }^{1}$ Ml Biologie Santé, Université Paris-Saclay, 91405 Orsay, \\ France \\ ${ }^{2}$ Institut de chimie physique, UMR8000, \\ Université Paris-Saclay, 91405 Orsay, France. \\ margot.draveny@universite-paris-saclay.fr \\ assmaa.ghali@universite-paris-saclay.fr \\ oliver.nusse@universite-paris-saclay.fr
}

> L'infection de la muqueuse gastrique par la bactérie pathogène Helicobacter pylori est une cause majeure de gastrite, pouvant évoluer en ulcère gastro-duodénal [1], voire en cancer de l'estomac. L'infection par $H$. pylori attire des cellules immunitaires, notamment les granulocytes neutrophiles, qui ne parviennent cependant pas à éliminer la bactérie. Un facteur de virulence de $H$. pylori associé au développement de ces maladies est l'îlot de pathogénicité cag-PAI [2]. Celui-ci code un système de sécrétion de type IV (T4SS) ainsi que la toxine CagA (cytotoxin-associated gene $A$ ), qui est injectée dans la cellule cible via T4SS. La présence de CagA dans la cellule hôte entraîne une perturbation des voies de signalisation cellulaires qui a de nombreuses conséquences: modification de la morphologie des cellules épithéliales par remodelage de l'actine et perturbation des jonctions serrées intercellulaires, induction d'interleukine 8, ou encore, inhibition de l'apoptose [3].

Quels facteurs des cellules hôtes permettent la translocation de CagA ? $H$. pylori interagit avec l'intégrine $\beta 1$, mais cette interaction n'est pas nécessaire pour la translocation de CagA [4]. Par ailleurs, la bactérie cible différentes molécules de la famille des carcinoembryonic antigen-related cell adhesion molecules (CEACAM) $[5,6]$. Ces molécules appartiennent à la super-famille des $\operatorname{IgCAM}^{1}$ et sont situées à la surface des cellules hôtes. H. pylori se sert de ces

${ }^{1}$ Glycoprotéines membranaires impliquées dans l'adhérence entre cellules. molécules (CEACAM1, 3, 5 et 6) comme récepteurs pour se fixer à la cellule cible via sa protéine de membrane externe HopQ. Cette interaction permet la translocation de CagA dans les cellules épithéliales de l'estomac [5-7]. Jusqu'à présent, aucune étude n'avait exploré le rôle et les conséquences de l'interaction HopQ-CEACAM dans le cadre de l'interaction de $H$. pylori et des cellules du système immunitaire. Or, les CEACAMl et 6 sont exprimées par toutes les cellules myéloïdes tandis que l'expression de CEACAM3 est restreinte aux granulocytes neutrophiles.

Récemment, Behrens et coll. ont démontré l'importance de l'interaction HopQCEACAM lors d'une infection par $H$. pylori et les effets de cette interaction sur le fonctionnement cellulaire des granulocytes neutrophiles [8].

\section{L'interaction HopQ-CEACAM permet la translocation et la phosphorylation de CagA dans les granulocytes neutrophiles}

Les chercheurs ont utilisé comme modèle d'étude des granulocytes neutrophiles murins exprimant des récepteurs CEACAM humains (hCEACAMl ou hCEACAM3/6 ou h(EACAM all) issus de souris transgéniques. H. pylori n'interagissant qu'avec les récepteurs humains, des granulocytes neutrophiles humains et des granulocytes neutrophiles murins ont été utilisés respectivement comme témoins positifs et négatifs de l'interaction.

Après s'être assurés de l'expression des molécules humaines CEACAM à la surface des granulocytes neutrophiles murins, les chercheurs ont mis en évidence le rôle de la protéine bactérienne HopQ dans l'interaction de $H$. pylori avec les granulocytes neutrophiles, par des expériences de cytométrie en flux. L'utilisation de souches d'H. pylori exprimant ou n'exprimant pas HopQ, a permis de montrer que l'expression de cette protéine était une condition nécessaire à de fortes interactions entre la bactérie et les granulocytes neutrophiles.

L'interaction HopQ-CEACAM permettant la translocation et la phosphorylation de la protéine CagA dans les cellules épithéliales de l'estomac, les chercheurs ont étudié ce mécanisme dans les granulocytes neutrophiles. Deux formes de la protéine CagA phosphorylée ont été détectées par une analyse en western blot, dont une correspond à sa forme clivée. Contrairement aux granulocytes humains, dans lesquels seule la forme clivée apparaît, les deux formes sont observées dans les granulocytes murins humanisés. Les chercheurs ont alors fait I'hypothèse que le clivage de CagA se produit moins rapidement dans ces derniers. Pour prouver que CagA est bien transloquée dans les granulocytes, les auteurs ont alors exprimé dans les bactéries une $\beta$-lactamase fusionnée à CagA. Un composé fluorescent intracellulaire est alors détecté par cytométrie en flux après son clivage par l'enzyme. Les résultats obtenus montrent un fort taux de translocation de CagA, en accord avec les résultats de l'analyse par western blot.

Après avoir montré l'importance de l'interaction HopQ-CEACAM pour la translocation et la présence de la forme active phosphorylée de CagA dans leurs expé- 


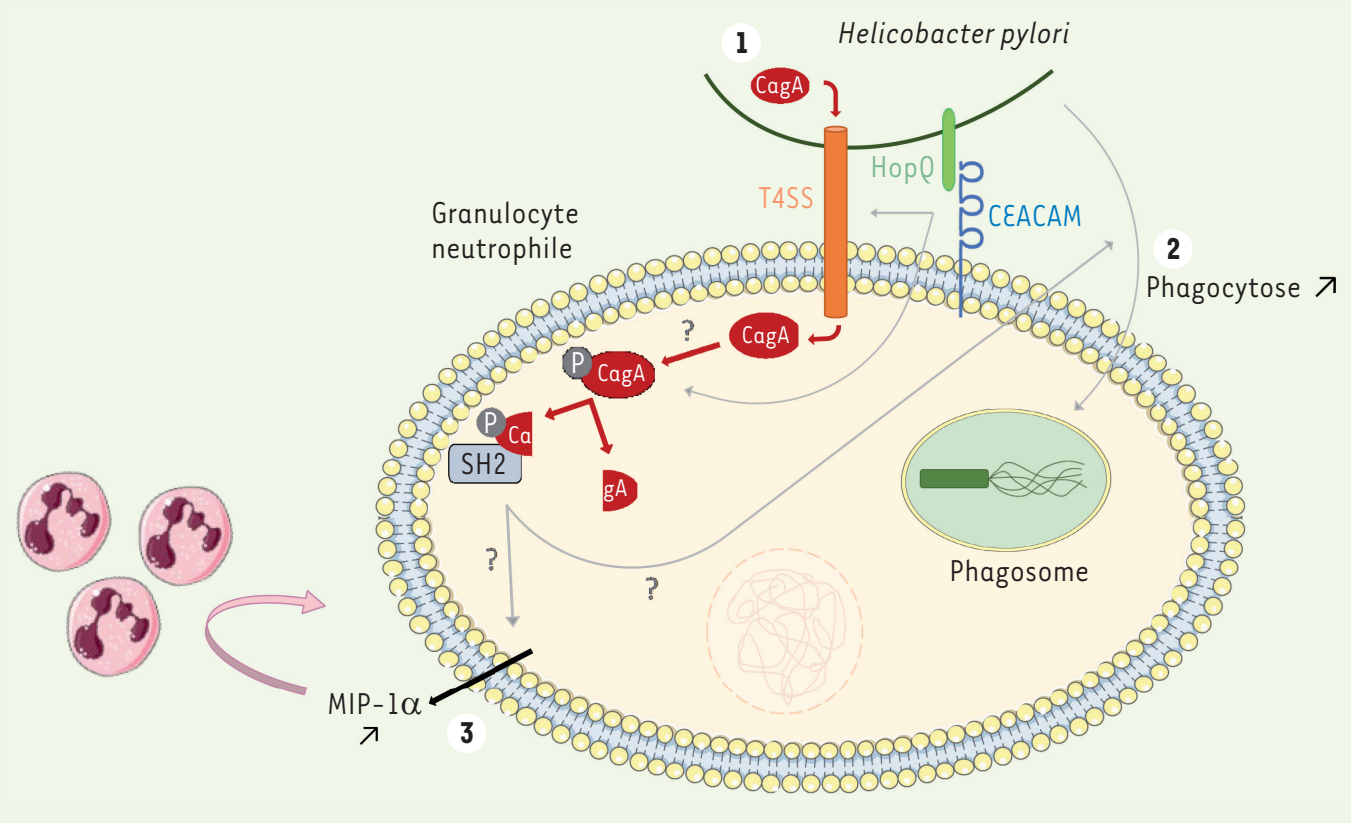

Figure 1. L'interaction Hopl-CEACAM entre H. pylori et le granulocyte neutrophile favorise la translocation de la protéine bactérienne CagA dans le granulocyte via le système de sécrétion bactérien T4SS (1). Une fois transloquée, CagA est phosphorylée puis clivée, et interagit avec des protéines à domaine SH2. Celles-ci modifient la signalisation cellulaire, une modification pouvant être responsable de l'augmentation de la phagocytose de la bactérie (2) et de l'augmentation de la sécrétion de la chimiokine MIP-l $\alpha$ (3), dont le rôle est de recruter d'autres granulocytes neutrophiles.

riences in vitro, les chercheurs ont testé la pertinence de cette interaction in vivo. Ils ont comparé des granulocytes neutrophiles humanisés provenant de souris infectées par $H$. pylori, soit de façon chronique pendant 4 semaines, soit pour la première fois (souris «naïves »). Une diminution significative de la translocation de CagA et de l'expression des récepteurs hCEACAM est observée dans les granulocytes des souris infectées de façon chronique comparativement à ceux des souris naïves.

Ces résultats confirment l'importance de l'interaction HopQ-CEACAM pour la translocation de CagA in vivo. De plus, ils révèlent une diminution des récepteurs CEACAM à la surface des cellules lors d'une infection persistante par H. pylori.

\section{Conséquences d'une infection par H. pylori pour les granulocytes neutrophiles}

Afin de déterminer si la présence de molécules CEACAM humaines à la surface des granulocytes murins a un impact sur le fonctionnement cellulaire lors de l'infection, la concentration de chimiokines sécrétées par les cellules murines exprimant les récepteurs humains a été mesurée et comparée à celle de cellules murines ne les exprimant pas. Les chercheurs ont réalisé un test immunologique permettant de détecter trois chimiokines: MIP- $1 \alpha(C C L 3)$ et $K C(C X C L 1)$, impliquées dans le recrutement et l'activation des granulocytes neutrophiles, et MCP-1 (CCL2) qui recrute les monocytes et contrôle la migration des macrophages. Les résultats ont montré que seule MIP-l $\alpha$ est davantage sécrétée par les granulocytes murins humanisés que par les granulocytes non humanisés.

Enfin, les chercheurs ont évalué les conséquences physiologiques liées à l'infection par $H$. pylori sur des granulocytes murins humanisés. Ils ont observé, par microscopie confocale, la phagocytose de la bactérie, qu'ils ont ensuite quantifiée par cytométrie en flux. Cette analyse a révélé la présence de nombreuses bactéries intracellulaires dans les granulocytes humanisés, mais pas dans les granulocytes non humanisés, soulignant l'importance de l'interaction HopQ-CEACAM. Par ailleurs, la présence de récepteurs hCEACAM3/6 s'est révélé augmenter la capacité de $H$. pylori à survivre dans le phagosome ${ }^{2}$. Pour finir, des expériences de cytométrie en flux ont montré une production de formes réactives de l'oxygène plus importante lorsque $H$. pylori interagit avec des granulocytes exprimant hCEA$\mathrm{CAM} 3 / 6$ que lorsque la bactérie interagit avec des granulocytes neutrophiles exprimant hCEACAMI. La raison de cette différence reste à comprendre. Les résultats d'une étude plus ancienne suggèrent que l'augmentation des formes réactives de l'oxygène est extracellulaire, expliquant la survie de H. pylori dans le phagosome [9].

\section{Les conséquences multiples}

\section{de l'interaction HopQ-CEACAM}

L'interaction HopQ-CEACAM de H. pylori avec les granulocytes neutrophiles permet la translocation de la toxine

\footnotetext{
${ }^{2}$ Le phagosome est un organite formé dans une cellule phagocytaire à la suite de la phagocytose.
} 
CagA dans ces cellules via le système de sécrétion T4SS de la bactérie. Après translocation, CagA est phosphorylée puis clivée. La raison de ce clivage est inconnue à ce jour, et le rôle de CagA dans les cellules immunitaires reste à étudier. Dans les cellules humaines, plusieurs protéines possédant un domaine SH2 (Src homology 2) sont connues pour se lier à la protéine CagA phosphorylée. Ces protéines modifient la signalisation cellulaire et seraient responsables de la modification du fonctionnement cellulaire, notamment de la phagocytose de H. pylori, et de la sécrétion de la chimiokine MIP-l $\alpha$, dont le rôle est de recruter d'autres granulocytes neutrophiles, contribuant ainsi à augmenter la réponse inflammatoire, ce qui serait à l'origine de la gastrite (Figure 1). De plus, il semblerait que $H$. pylori soit capable de moduler sa survie dans le phagosome, peut-être en détournant la NADPH oxydase vers la membrane plasmique, induisant une production de formes réactives de l'oxygène vers l'extérieur de la cellule, ce qui pourrait contribuer aux lésions gastriques. Quant à la diminution de l'expression des CEACAM sur les granulocytes neutrophiles lors d'une infection chronique, elle pourrait être due aux granulocytes eux-mêmes, ou à la bactérie, afin de limiter les dommages causés par l'infection et assurer ainsi sa survie. $\diamond$ The HopQ-CEACAM interaction controls CagA translocation, phosphorylation, and phagocytosis of Helicobacter pylori in neutrophilic granulocytes

\section{LIENS D'INTÉRÊT}

Les auteurs déclarent n'avoir aucun lien d'intérêt concernant les données publiées dans cet article.

\section{RéFÉRENCES}

1. Suerbaum S, Michetti P. Helicobacter pylori infection. N Engl J Med 2002 ; 347 : 1175-86.

2. Covacci $A$, Censini $S$, Bugnoli $M$, et al. Molecular characterization of the $128-\mathrm{kDa}$ immunodominant antigen of Helicobacter pylori associated with cytotoxicity and duodenal ulcer. Proc Natl Acad Sci USA 1993 ; $90: 5791-5$.
3. Backert S, Tegtmeyer N, Selbach M. The versatility of Helicobacter pylori CagA effector protein functions: The master key hypothesis. Helicobacter $2010 ; 15$ : 163-76.

4. Zhao $Q$, Busch B, Jimenez-Soto LF, et al. Integrin but not CEACAM receptors are dispensable for Helicobacter pylori CagA translocation. PLoS Pathog 2018; 14 : e1007359.

5. Königer V, Holsten L, Harrison U, et al. Helicobacter pylori exploits human CEACAMs via Hop $\mathrm{f}$ for adherence and translocation of CagA. Nat Microbiol 2016 ; 2 : 16188-99.

6. Javaheri A, Kruse T, Moonens K, et al. Helicobacter pylori adhesin Hop $Q$ engages in a virulence-enhancing interaction with human CEACAMs. Nat Microbiol 2017 ; 2: 16189 .

7. Belogolova $\varepsilon$, Bauer B, Pompaiah M, et al. Helicobacter pylori outer membrane protein HopQ identified as a novel T4SS-associated virulence factor. Cell Microbiol 2013 ; 15 : 1896-1912.

8. OBehrens IK, Busch B, Ishikawa-Ankerhold $\mathrm{H}$, et al. The HopQ-CEACAM interaction controls CagA translocation, phosphorylation, and phagocytosis of Helicobacter pylori in neutrophils. mBio 2020; 11 : e03256-19.

9. Allen LA, McCaffrey RL. To activate or not to activate: distinct strategies used by Helicobacter pylori and Francisella tularensis to modulate the NADPH oxidase and survive in human neutrophils. Immunol Rev 2007 ; 219: 103-17

P ossédées du Malin au Moyen-Âge, les sorcières hystériques sont vouées au bûcher. Enfermées au XvII siècle, maltraitées, elles rejoignent la Cour des Miracles de l'Hospice de la Vieillesse-Femmes à la Salpêtrière... Jusqu'à ce que le Dr JeanMartin Charcot (1825-1893) mène le combat qui transforme l'ancien hospice en hôpital : l'École de la Salpêtrière de

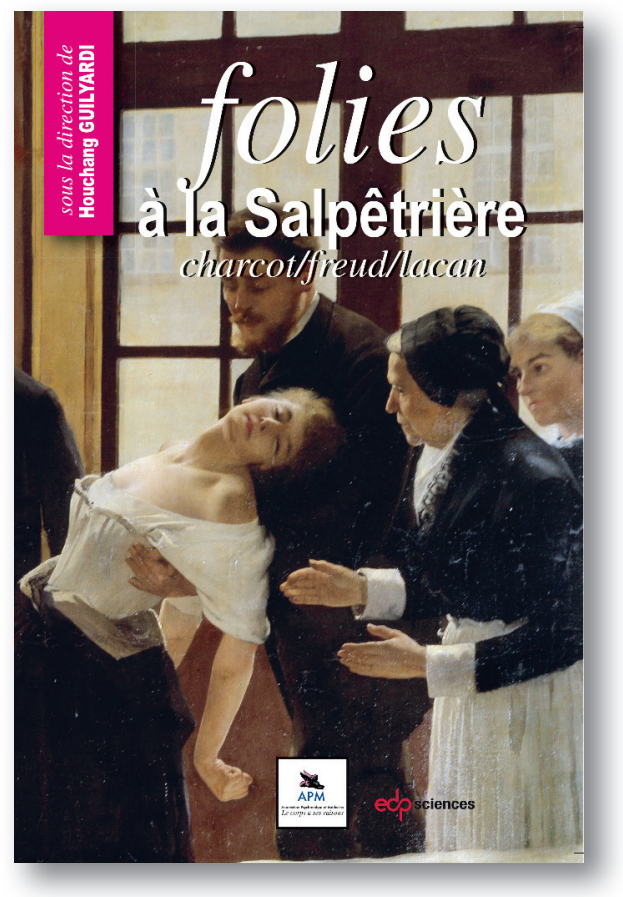

ISBN : 978-2-7598-1268-4
240 pages

$20 €$ Paris est née, qui devient lieu de recherche, d'enseignement et de soins, de renommée internationale.

Jean Martin Charcot n'a pas bonne presse, et pourtant... Hystérie et folie traversent les siècles, prenant les formes de «l'air du temps ».

De l'utérus migrateur d'Hippocrate aux recherches neurologiques de Charcot. Du désir inconscient avec Freud à la jouissance du parlêtre chez Lacan... C'est à cette traversée historique et conceptuelle que nous convie cet ouvrage.

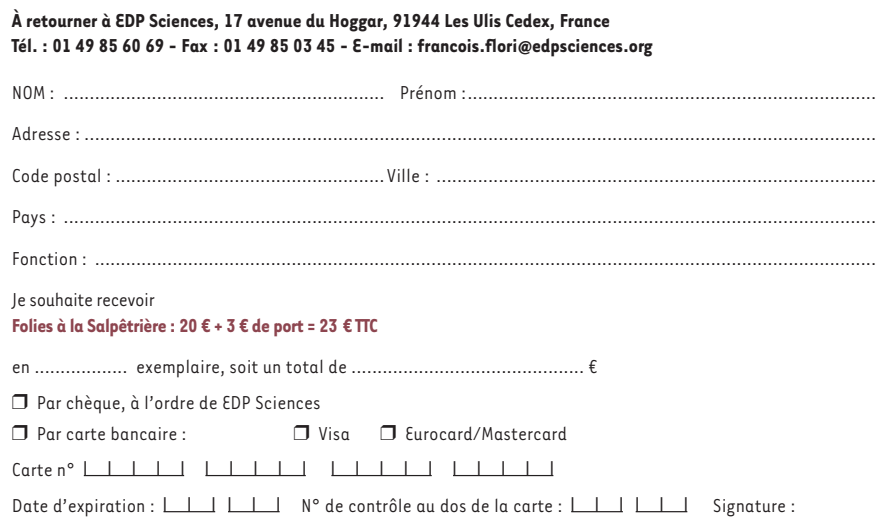

